\title{
Avaliação e características das quedas de pacientes durante a internação hospitalar
}

\section{Evaluation and Characteristics of Falls of Patients during Hospitalization}

\author{
Evaluación y características de caídas de pacientes durante la hospitalización
}

\author{
Cristian Dornelles ${ }^{1}$, ORCID 0000-0001-9002-613X \\ Joycianne Ramos Vasconcelos de Aguiar ${ }^{2}$, ORCID 0000-0002-0203-4939 \\ Mariana Bonati de Matos ${ }^{3}$, ORCID 0000-0003-1196-7228 \\ Lidiane Aguiar Ferreira ${ }^{4}$, ORCID 0000-0003-3343-318X \\ Susana Cecagno ${ }^{5}$, ORCID 0000-0002-3953-0688 \\ Athaynne Ramos de Aguiar Prado ${ }^{6}$, ORCID 0000-0001-7617-3969 \\ $123{ }^{4}$ Universidade Católica de Pelotas, Brasil \\ ${ }^{5}$ Universidade Federal de Pelotas, Brasil \\ ${ }^{6}$ Universidade Federal do Estado do Rio de Janeiro, Brasil
}

Resumo: Objetivo: Avaliar e caracterizar as quedas de pacientes em um hospital no sul do Brasil. Método: Estudo descritivo e retrospectivo de 73 notificações de quedas em um hospital no sul do Brasil, de janeiro 2016 a dezembro de 2019. Utilizou-se dados secundários obtidos pelo Núcleo de Segurança do Paciente da instituição e da Morse Fall Scale, sendo a análise estatística descritiva. Resultados: A maioria dos pacientes que sofreram quedas foi do sexo masculino $(60,3 \%),>61$ anos $(50,6 \%)$, tratamento clínico $(89,0 \%)$, portadores de neoplasias $(43,8 \%)$ em uso contínuo de medicações $(91,8 \%)$. As quedas ocorreram da própria altura $(67,1 \%)$, no banheiro $(56,2 \%)$ e no turno da noite $(50,0 \%)$. Em $50,7 \%$ das quedas houve danos ao paciente, sendo os danos considerados como: leve $(62,2 \%)$, moderado $(32,4 \%)$ e grave $(5,4 \%)$. Conclusão: Melhorar a compreensão das quedas e as suas consequências pode auxiliar os profissionais na avaliação dos riscos e no estabelecimento de medidas preventivas.

Palavras-chave: acidentes por quedas; pacientes internados; segurança do paciente; dano ao paciente; hospital.

Abstract: Objective: Evaluate and characterize the patients falls in a hospital in Southern Brazil. Method: Descriptive and retrospective study of 73 reports of falls in a hospital in Southern Brazil, from January 2016 to December 2019. Secondary data obtained by the institution's Patient Safety Center and the Morse Fall Scale were used, in a descriptive statistical analysis. Results: Most of the patients who suffered falls were male $(60.3 \%),>61$ years old $(50.6 \%)$, clinical treatment $(89.0 \%)$, patients with neoplasms $(43.8 \%)$ in continuous medication use $(91.8 \%)$. The falls occurred from their own same height $(67.1 \%)$, 
in the bathroom (56.2\%) and in the night shift (50.0\%). In 50.7\% of the falls there was damage to the patient, mild damage $(62.2 \%)$, moderate damage $(32.4 \%)$ and severe damage (5.4\%). Conclusion: Improving the understanding of falls and their consequences can help health professionals in assessing risks and establishing preventive measures.

Keywords: accidental falls; inpatients; patient safety; patient harm; hospital.

Resumen: Objetivo: Evaluar y caracterizar las caídas de pacientes en un hospital del sur de Brasil. Método: Estudio descriptivo y retrospectivo de 73 notificaciones de caídas en un hospital del sur de Brasil, de enero de 2016 a diciembre de 2019. Se utilizaron datos secundarios obtenidos por el Núcleo de Seguridad del Paciente de la institución y la Escala de Caídas Morse, con análisis estadístico descriptivo. Resultados: La mayoría de los pacientes que sufrieron caídas fueron hombres $(60,3 \%),>61$ años $(50,6 \%)$, tratamiento clínico $(89,0 \%)$, portadores de neoplasias $(43,8 \%)$ en uso de medicación continua $(91,8 \%)$. Las caídas ocurrieron desde la misma altura $(67,1 \%)$, en el baño $(56,2 \%)$ y en el turno de la noche $(50,0 \%)$. En el 50,7\% de las caídas hubo daño al paciente, considerados como: ligero $(62,2 \%)$, moderado $(32,4 \%)$ y grave $(5,4 \%)$. Conclusión: Mejorar la comprensión de las caídas y sus consecuencias puede ayudar a los profesionales de la salud en la evaluación de los riesgos y en el establecimiento de medidas preventivas.

Palabras claves: accidentes por caídas; pacientes internados; seguridad del paciente; daño del paciente; hospital.

Recebido: 20/03/2021

Aceito: $29 / 10 / 2021$

Como citar:

Dornelles C, De Aguiar JRV, De Matos MB, Ferreira LA, Cecagno S, Prado ARdA. Avaliação e características das quedas de pacientes durante a internação hospitalar. Enfermería: Cuidados Humanizados. 2021;10(2):160-174. DOI: 10.22235/ech.v10i2.2499

Correspondência: Cristian Dornelles. E-mail: dornelles.cristian@gmail.com

\section{Introdução}

A queda é conceituada como um episódio em que a pessoa cai ao chão ou em um nível mais baixo do que a sua posição inicial, de forma súbita e não intencional, sem perder a consciência. ${ }^{(1)}$ Estima-se que a cada ano 646.000 quedas fatais ocorrem no mundo, fazendo delas a segunda maior causa de morte não intencional, perdendo, apenas, para os acidentes de trânsito. (2)

Em 2004, a Organização Mundial da Saúde (OMS) procurou organizar os conceitos e as definições sobre a segurança do paciente, desenvolvendo, com isso, a Classificação Internacional de Segurança do Paciente (International Classification for Patient Safety ICPS). Dentre os conceitos-chave, destacam-se: incidente - evento ou circunstância que poderia ter resultado, ou resultou, em dano desnecessário ao paciente; evento adverso incidente que resulta em dano ao paciente; dano - comprometimento da estrutura ou função do corpo e/ou qualquer efeito dele oriundo, incluindo-se doenças, lesão, sofrimento, morte, incapacidade ou disfunção, podendo, assim, ser físico, social ou psicológico. ${ }^{(3)}$ 
Na área da saúde, as quedas são consideradas eventos adversos, isso é, incidentes que resultam em potenciais danos à saúde, embora, sejam preveníveis. Nos hospitais, as quedas constituem $70 \%$ dos eventos adversos notificados. Desses eventos intra-hospitalares, 30\% podem resultar em lesões físicas e 4 a $6 \%$, em lesões graves. ${ }^{(4)}$

No Brasil, as quedas representam o segundo evento adverso mais notificado e o segundo mais relacionado a óbitos, conforme o Sistema Notivisa da Agência Nacional de Vigilância Sanitária, que registrou 36.452 notificações de quedas entre março de 2016 até junho de 2019. ${ }^{(5)}$

No hospital, as quedas são eventos complexos e multifatoriais, apresentando origem intrínseca e extrínseca, que devem ser, criteriosamente, contextualizados e relacionados com as características individuais de cada paciente. Pode-se definir como fatores intrínsecos as alterações fisiológicas, que surgem com o envelhecimento (deficiência visual e/ou auditiva); alterações patológicas; fatores psicológicos; déficit cognitivo e fraqueza muscular. Em contrapartida, os fatores extrínsecos são ocasionados pela interação do indivíduo com o meio ambiente, como: a qualidade do piso, a iluminação precária, a falta de corrimão, a presença de grades laterais nas camas ou a ausência dessas, a existência de obstáculos no caminho, a ausência ou o auxílio técnico inadequado durante a locomoção, dentre outros fatores. ${ }^{(6)}$

Agir precocemente avaliando o risco que o paciente tem de cair, pode ser uma estratégia possível para atuar na prevenção, podendo ser feito por escalas que mensuram esses riscos durante a internação e que fornecem subsídios para os profissionais realizarem uma avaliação sistemática. Permite-se, assim, ações de prevenção, promoção e controle das quedas no ambiente hospitalar, individualmente, de acordo com o grau de risco préestabelecido. ${ }^{(7)}$

Dentre esses instrumentos/escalas, ressalta-se a Morse Fall Scale (MFS), ${ }^{(8)}$ traduzida e adaptada para a Língua Portuguesa em 2013, utilizando a sigla MFS-B. ${ }^{(9)}$

A internação hospitalar eleva, consideravelmente, o risco de queda, pois o paciente se encontra fora do seu ambiente familiar e do seu cotidiano, proporcionando que se mude as suas rotinas. Essas modificações, associadas ao estresse, podem agravar patologias prévias, como a demência, alterações relacionadas à visão e mobilidade física. As doenças agudas e o uso de vários fármacos associados também podem influenciar no risco de quedas e no agravamento do dano decorrente, prolongando a internação e aumentando os custos assistenciais, além de proporcionar ansiedade nos profissionais e perda da confiança na equipe e no hospital. ${ }^{(7)}$

Entende-se, assim, que é de extrema relevância que os gestores e os profissionais de saúde conheçam a realidade dos casos de quedas no seu local de trabalho, obtendo, dessa forma, subsídios para a criação de estratégias que estimulem a prevenção/diminuição desse evento na instituição hospitalar. ${ }^{(10)}$

Diante desse cenário, a questão norteadora definida para esse estudo foi: quais as principais características dos pacientes que sofreram quedas em um hospital no sul do Brasil?

A partir desse questionamento, o presente artigo tem como objetivo avaliar e caracterizar as quedas de pacientes em um hospital no sul do Brasil. 


\section{Metodologia}

Trata-se de um estudo descritivo, com abordagem quantitativa, de casos incidentes, realizados em um hospital no sul do Brasil, que ocorreu no período de janeiro/2016 a dezembro/2019.

O estudo é composto por todos os casos notificados de quedas de pacientes $(n=73$ notificações), registrados num sistema informatizado dos hospitais federais universitários, denominado VIGIHOSP, vinculado ao Núcleo de Segurança do Paciente da instituição. O sistema é de acesso público a todos os funcionários, que podem realizar a notificação da queda em qualquer momento, pelo acesso à página digital do hospital na internet. A notificação no sistema procura descrever as características da queda, o paciente, o motivo e as condutas adotadas após o incidente. A adesão à ferramenta de notificação tem aumentado durante as capacitações internas do hospital, mas se sabe que ainda existem subnotificações dos registros de quedas, visto que muitos profissionais acham que a notificação é algo punitivo. Na verdade, o trabalho empenhado tem a visão de uma cultura educativa. Foram incluídos na pesquisa os registros de incidentes de pacientes com idade acima de 18 anos, sendo excluídos os pacientes que não tiveram o preenchimento da escala de Morse Fall Scale pela equipe de saúde.

O estudo ocorreu em um hospital no sul do Brasil, que presta atendimento a 28 municípios, possuindo 175 leitos distribuídos nas seguintes áreas: clínica médica e especialidades clínicas, ginecologia e obstetrícia, pediatria, cirurgia geral e especialidades cirúrgicas, além de UTI Geral e UTI Neonatal. Para a realização desta pesquisa não foram envolvidos pacientes da UTI Neonatal e pediatria, visto que o estudo procurou apenas trabalhar com pacientes maiores de 18 anos.

Os dados foram coletados de forma retrospectiva, por dados secundários das notificações de eventos adversos relacionados à queda, recebidas pelo Núcleo de Segurança do Paciente (NSP) da instituição, além de contar com o preenchimento pela equipe de saúde da Morse Fall Scale, que faz avaliação do risco de queda que o paciente hospitalizado está sujeito.

Para cada paciente registrado foi preenchida uma ficha, contendo variáveis de dados sociodemográficos e clínicos dos pacientes, como: sexo, idade, unidade funcional do hospital, doença que motivou a internação, uso contínuo ou não de medicações, medicações prescritas no dia da ocorrência da queda.

As características relacionadas à ocorrência das quedas também foram mencionadas, como: turno da ocorrência da queda (considerando como manhã o período das $07 \mathrm{~h} 01 \mathrm{~min}$ às $13 \mathrm{~h}$, a tarde foi considerado o período das $13 \mathrm{~h} 01 \mathrm{~min}$ às $19 \mathrm{~h}$ e a noite das $19 \mathrm{~h} 01 \mathrm{~min}$ às $07 \mathrm{~h}$ ), local da ocorrência da queda, detalhe da queda (cama, poltrona, cadeira higiênica, cadeira de rodas, maca de transporte ou de própria altura, que se caracteriza como do mesmo nível de altura, não possuindo nenhum nível elevado), se o incidente foi presenciado ou não, quem presenciou a queda, houve relação com falta de equilíbrio, perda de força ou desorientação do paciente e se a queda teve ou não relação com problemas de infraestrutura da instituição. Nessa ficha também se procurou relatar as características dos danos gerados pelas quedas, relacionando-se as seguintes variáveis: houve ou não dano ao paciente e qual foi o tipo de dano ocasionado.

Por fim, foram relacionadas às medidas preventivas adotadas contra a ocorrência de quedas, pelas seguintes variáveis: avaliação do risco de queda, risco identificado, uso de pulseira de identificação de risco, descumprimento das orientações pelo paciente e familiares. Procurou-se relacionar quais as condutas adotadas após a ocorrência da queda. 
Nos casos em que o evento foi notificado por mais de um profissional, foi considerado apenas um evento e todos os profissionais que o notificaram.

Foi utilizada a Morse Fall Scale traduzida e adaptada para o português (MFS-B), ${ }^{(9)}$ que contém seis itens de avaliação e suas respectivas pontuações. A soma das pontuações de cada item gera um escore para a classificação em Risco Baixo (0-24 pontos), Risco Moderado (25-44 pontos) e Risco Elevado ( $\geq 45$ pontos). A análise da consistência interna da MSF-B revelou um Alpha de Cronbach baixo $(\alpha=0,28)$ na correlação entre os itens. A escala original possui seis itens, independentes uns dos outros, e medem informações diferenciadas. Essa é uma característica necessária para verificar um fenômeno multifacetado. Esse aspecto também foi encontrado na versão original $(\alpha=0,16)$ e na chinesa $(\alpha=0,26){ }^{(11)}$

A Morse Fall Scale está padronizada nesta instituição hospitalar desde janeiro de 2016, passando por processo de capacitação de todos os profissionais de saúde, desde o ano de 2015, sendo aplicada diariamente. Qualquer profissional de saúde capacitado poderá realizar o seu preenchimento e fornecer as devidas orientações, porém, na prática da instituição do estudo, a escala é preenchida pelo enfermeiro da unidade assistencial. Para a pesquisa, levou-se em conta o escore e o risco de queda do dia anterior ao ocorrido incidente notificado.

A classificação adotada para os tipos de dano ocasionado ao paciente foi: ${ }^{(12)}$ leve quando apresenta sintomas leves, com danos mínimos, perda de função, porém, com curta duração e com pequenas intervenções quando necessário; moderado quando o paciente é sintomático, necessita de intervenção, prolongando a internação, ocorrendo a perda de função permanente ou em longo prazo e grave quando o paciente é sintomático e necessita de intervenção para suporte de vida ou intervenção clínica/cirúrgica, ocasionando grande dano, perda de função permanente ou em longo prazo ou quando tem óbito associado.

A análise estatística descritiva foi realizada através do Statistical Package for the Social Sciences (SPSS), versão 26.0, por meio de freqüências absolutas e relativas. A pesquisa foi aprovada pelo Comitê de Ética. Por se tratar da coleta de dados secundários, não foi necessária a aplicação do Termo de Consentimento Livre e Esclarecido e não houve a identificação dos participantes, conforme a resolução 466/2012 do Conselho Nacional de Saúde.

\section{Resultados}

Foram avaliados entre janeiro de 2016 a dezembro de 2019 os dados de 73 notificações de quedas durante a internação. A média de internações hospitalares durante estes quatro anos foi de 4.438. Dos pacientes que sofreram queda, $44(60,3 \%)$ são homens, possuem 60 anos ou mais $(50,6 \%), 65(89,0 \%)$ estavam internados para tratamento clínico e $67(91,8 \%)$ estavam realizando uso contínuo de medicações (Tabela 1). 
Tabela 1. Características dos pacientes internados com ocorrência de quedas em um hospital no sul do Brasil. Brasil, 2020

\begin{tabular}{|c|c|c|}
\hline Variáveis & $\mathbf{N}$ & $\%$ \\
\hline \multicolumn{3}{|l|}{ Sexo } \\
\hline Masculino & 44 & 60.3 \\
\hline Feminino & 29 & 39.7 \\
\hline \multicolumn{3}{|l|}{ Idade } \\
\hline $20-40$ anos & 14 & 19.2 \\
\hline $41-59$ anos & 21 & 28.8 \\
\hline 60 anos ou mais & 37 & 50.6 \\
\hline \multicolumn{3}{|c|}{ Doença que motivou a internação } \\
\hline Oncológica & 32 & 43.8 \\
\hline Infectologia & 12 & 16.4 \\
\hline Respiratória & 12 & 16.4 \\
\hline Gastrointestinal & 08 & 11.0 \\
\hline Endócrina & 05 & 6.8 \\
\hline Ginecológica/Obstétrica & 02 & 2.8 \\
\hline Outras & 02 & 2.8 \\
\hline \multicolumn{3}{|c|}{ Medicações prescritas no dia da queda } \\
\hline Ansiolíticos & 41 & 56.2 \\
\hline Anti-hipertensivos & 36 & 49.3 \\
\hline Antidepressivos & 34 & 46.6 \\
\hline Anti-diabéticos & 25 & 34.2 \\
\hline Diuréticos & 24 & 32.9 \\
\hline Anticoagulantes & 21 & 28.8 \\
\hline Antipsicóticos & 18 & 24.7 \\
\hline
\end{tabular}

Fonte: Criação do autor, 2020.

Com relação às características das quedas, constatou-se que $30(50,0 \%)$ das quedas ocorreram durante o turno da noite e em $51(69,9 \%)$ dos casos a queda foi presenciada por um profissional de saúde ou familiar. No que se refere aos problemas de infraestrutura da instituição hospitalar, $57(78,1 \%)$ das quedas não apresentaram relação com este aspecto (Tabela 2). 
Tabela 2. Características relacionadas à ocorrência das quedas em um hospital no sul do Brasil. Brasil, 2020

\begin{tabular}{lcc}
\hline Variáveis & N & \% \\
\hline Local da ocorrência da queda & 41 & 56.2 \\
Banheiro & 15 & 20.5 \\
Leito hospitalar & 10 & 13.7 \\
Corredores & 07 & 9.6 \\
Outros & & \\
Detalhe do local da queda & 49 & 67.1 \\
Própria altura & 11 & 15.1 \\
Cama & 07 & 9.6 \\
Cadeira higiênica & 02 & 2.7 \\
Poltrona & 02 & 2.7 \\
Cadeira de rodas & 02 & 2.7 \\
Maca de transporte & & \\
Falta de equilíbrio do paciente & 52 & 71.2 \\
Sim & 21 & 28.8 \\
Não & & \\
Perda de força do paciente & 40 & 54.8 \\
Não & 33 & 45.2 \\
Sim & & \\
Desorientação do paciente & 52 & 71.2 \\
Não & 21 & 28.8 \\
Sim & & \\
\hline
\end{tabular}

Fonte: Criação do autor, 2020.

O estudo constatou que em $37(50,7 \%)$ das quedas ocorreram danos à saúde do paciente. A ocorrência de danos leves foi de $23(62,2 \%)$ casos, observado em situações que o paciente apresentou escoriações de pele, seguido dos danos moderados $12(32,4 \%)$, caracterizados por ferimentos cortocontuso em quatro pacientes $(33,4 \%)$ e traumatismo crânioenfelálico leve em oito pacientes $(66,6 \%)$. Os danos graves corresponderam a dois $(5,4 \%)$ de todos os incidentes gerados. A queda de um paciente culminou na fratura de nariz e no outro na fratura de punho (Tabela 3 ).

Tabela 3. Características dos danos aos pacientes decorrentes das quedas no período de janeiro 2016 a dezembro de 2019. Brasil, 2020

\begin{tabular}{lcc}
\hline Variáveis & $\mathbf{N}$ & \% \\
\hline Tipo de dano* $(\mathbf{n = 3 7})$ & 23 & 62.2 \\
Leve & 12 & 32.4 \\
Moderado & 02 & 5.4 \\
Grave & \\
\hline
\end{tabular}

*somente pacientes que sofreram danos.

Fonte: Criação do autor, 2020. 
No que se refere à avaliação do risco de queda, 60 (82,2\%) foram identificados com risco de queda, sendo 21 (35,0\%) classificados com risco moderado, conforme a Morse Fall Scale no dia anterior à ocorrência da queda. Nas avaliações diárias da Morse Fall Scale, o enfermeiro, além de preencher a pontuação correspondente, orienta os pacientes e os familiares sobre as medidas preventivas, conforme o protocolo de risco de queda da instituição, que menciona sobre manter as grades das camas elevadas durante todo o período, utilização da luz de cabeceira durante a noite, de que toda saída do leito deve ser avisada a enfermagem, dentre outras informações. As medidas preventivas adotadas contra a ocorrência de quedas no hospital estão caracterizadas na Tabela 4.

Tabela 4: Características relacionadas às medidas preventivas contra a ocorrência de quedas. Brasil, 2020

\begin{tabular}{lll}
\hline Variáveis & N & \% \\
\hline Qual é o risco identificado* (n=60) & & \\
Sem risco & 09 & 15.0 \\
Risco baixo & 17 & 28.3 \\
Risco moderado & 21 & 35.0 \\
Risco elevado & 13 & 21.7 \\
Uso de pulseira de identificação de risco & & \\
Não & 40 & 54.8 \\
Sim & 33 & 45.2 \\
Descumprimento das orientações pelo paciente/ familiar & & \\
Sim & 53 & 72.6 \\
Não & 20 & 27.4 \\
\hline
\end{tabular}

*apenas pacientes avaliados com risco de queda

Fonte: Criação do autor, 2020.

Após a ocorrência das quedas na instituição hospitalar em 60 (82,2\%) dos agravos, a única conduta tomada foi à avaliação médica, não se utilizando de nenhum exame de imagem como recurso auxiliar na investigação. A instituição utiliza de um protocolo de risco de queda, que menciona as condutas realizadas mediante a sua ocorrência, no qual cita que o profissional de saúde que se deparou com a queda, deve avaliar as condições clínicas após a queda e posicionar o paciente em condições seguras (sentado ou deitado), comunicar a equipe responsável para realizar o exame físico e solicitar avaliação médica. Ressalta-se que é de responsabilidade do médico assistencial providenciar os exames de imagem (raio-x ou tomografia), conforme avaliação inicial, realizar a reavaliação do paciente, orientar o paciente e acompanhante sobre o risco de queda, além de notificar o incidente no VIGIHOSP (Tabela 5). 
Tabela 5. Principais condutas adotadas após a ocorrência de quedas na instituição hospitalar, Brasil, 2020

\begin{tabular}{lcc}
\hline Variáveis & $\mathbf{N}$ & \% \\
\hline Conduta adotada após queda & & \\
Avaliação médica & 60 & 82.2 \\
Avaliação médica + Raio-x & 10 & 13.7 \\
Avaliação médica + Tomografia & 03 & 4.1 \\
\hline
\end{tabular}

Fonte: Criação do autor, 2020.

\section{Discussão}

O objetivo deste estudo foi avaliar e caracterizar as quedas de pacientes em um hospital no sul do Brasil. A maior parte da amostra era do sexo masculino (60,3\%). Corroborando com a literatura que apresenta resultados semelhantes, demonstrou-se que 53 a $63,7 \%$ de homens sofreram queda, relacionando esses dados a fatores culturais, como maior dificuldade em pedir e aceitar ajuda na execução das suas atividades diárias. ${ }^{(6,13-14)}$ Em contrapartida, alguns autores encontraram uma maior prevalência de quedas entre as mulheres e associaram a uma maior prevalência de doenças crônicas, maior incidência de osteoporose e alterações de ordem hormonal na pós-menopausa, fatores que interferem no equilíbrio postural, bem como na redução de massa muscular. ${ }^{(7,15)}$

Com relação à faixa etária, os pacientes que sofreram quedas apresentaram idade igual ou superior a 60 anos $(50,6 \%)$. Sabe-se que o avanço da idade desencadeia uma série de alterações fisiológicas, consideradas fatores de risco para a ocorrência de quedas, mais frequentes nessa faixa etária. Os fatores comumente observados são aqueles relativos à instabilidade postural, alteração da marcha, diminuição da cognição, déficits sensoriais, além dos agravos crônico-degenerativos. ${ }^{(6-7)}$

Os dados epidemiológicos apontam que cerca de um terço dos idosos sofre ao menos uma queda ao ano. ${ }^{(16)}$ As quedas cometem cerca de $30 \%$ dos idosos acima de 60 anos e de 40 a $50 \%$ dos idosos mais velhos (acima de 80 a 85 anos), consistindo na principal causa de lesões, fatais ou não, nesses grupos. ${ }^{(17)}$ Estes incidentes são considerados um problema de saúde pública, pois são mais frequentes na velhice e suas sequelas podem diminuir a independência funcional do idoso e aumentar o risco de morte precoce, ${ }^{(18)}$ além de mencionar que as internações por quedas implicam na sobrevida, pois apenas cerca de $50 \%$ dos idosos que caíram e foram admitidos em hospitais estarão vivos após um ano. ${ }^{(19)} \mathrm{A}$ quantidade de idosos acima de 60 anos cresce mundialmente a uma taxa acelerada de $3 \%$ ao ano, ampliando o número de pessoas em risco de quedas, e consequentemente com sequelas e fatalidades. No ano de 2017, a população mundial foi estimada em 7,6 bilhões de pessoas, $13 \%$ delas idosas. Para o ano de 2050, ela será de 9,8 bilhões de pessoas, das quais $21 \%$ serão idosas, no mesmo momento que o Brasil estará entre os 31 países mais populosos do mundo, os quais, em conjunto, concentrarão $75 \%$ da população global. ${ }^{(20)}$

O turno de maior ocorrência de quedas registradas foi o período da noite, o que vai ao encontro de grande parte dos estudos publicados. ${ }^{(14-15)} \mathrm{Na}$ prática diária do ambiente hospitalar, constata-se a tendência dos pacientes não recorrerem aos enfermeiros para auxiliar na realização de atividades que julgam capazes, como ir ao banheiro, o que pode agravar-se no período da noite, quando o quantitativo de profissionais nas unidades é menor, além disso devemos levar em consideração as mudanças sensoriais fisiológicas da velhice, visto que o a maior parte das quedas ocorreram com pacientes acima de 60 anos. 
Em relação ao local da queda, houve maior ocorrência nos setores clínicos, que correspondem, na instituição, à maioria dos leitos disponíveis, além de receberem pacientes de diversas especialidades. A alta incidência de quedas em unidades de internação clínica pode ser associada ao longo tempo de permanência do paciente, idade avançada e alta complexidade da assistência. ${ }^{(4,7)} \mathrm{O}$ fato de grande parte das patologias relacionadas às quedas neste estudo serem de origem oncológica, justificam-se devido à instituição em que a presente pesquisa foi realizada, que é um hospital referência para o município e as demais cidades vizinhas no tratamento das mais diversas doenças oncológicas.

Nesta pesquisa, a maioria das quedas notificadas foram da própria altura e ocorreram no banheiro. Estudos realizados em hospitais no sul do Brasil encontraram que esse tipo de queda foi a mais frequente e pode estar associado aos fatores intrínsecos do paciente, tais como desorientação, incapacidade cognitiva, fraqueza, uso de medicamentos e comorbidades. ${ }^{(6,7)}$

Sabe-se que tanto ao banheiro, como o próprio quarto/enfermaria são os locais mais utilizados pelos pacientes durante o período de internação hospitalar e é comum, portanto, que sejam apontados como os de maior ocorrência desse tipo de evento adverso. (13,15) Estudos demonstram que $50 \%$ das quedas podem ser evitadas com o aprimoramento da estrutura hospitalar, principalmente nas áreas de piso escorregadio, como banheiros, onde não há o uso de pisos antiderrapantes, nem a presença de barras de apoio em quantidade e altura ideais e muito menos há a presença de bancos nos boxes de chuveiros. ${ }^{(21)}$ Das 36.452 notificações de quedas ocorridas em todo o país e recebidas pela Anvisa, no período de março de 2014 a junho de 2019, 21.296 (58\%) delas ocorreram no quarto e no banheiro. ${ }^{(5)}$

É importante ressaltar que devido à queda ser considerada um evento de causas multifatoriais, uma única queda pode ter mais de um fator contribuinte. No nosso estudo, percebe-se o predomínio das causas relacionadas à falta de equilíbrio do paciente $(71,2 \%)$, perda de força $(45,2 \%)$ e desorientação mental $(28,8 \%)$. Nesse sentido, a presença de marcha fraca e/ou prejudicada foi identificada em $47 \%$ dos pacientes em um hospital americano que apresentaram queda; desses, $34 \%$ utilizavam dispositivos de assistência para deambular. ${ }^{(22)}$

Em relação à associação das quedas com problemas de infraestrutura, o estudo apresentou 16 quedas $(21,9 \%)$ com relação direta a problemas estruturais, como: iluminação precária, camas com a guarda danificada, pisos escorregadios, falta de barras auxiliares nos banheiros, dentre outros fatores. Na literatura foi encontrado um estudo realizado no hospital público de São Paulo em 2015, que relacionou 50\% das notificações de queda, com falta de interruptor de luz acessível e mobiliários sem rodas e travas de segurança, além da ausência de camas com grades em todos os leitos. Esse último quesito alerta para o fato de que $23 \%$ de todas as quedas notificadas ocorreram da cama ou leito. ${ }^{(23)}$

O uso contínuo de medicamentos mostrou associação com a ocorrência de quedas no ambiente hospitalar no nosso estudo, sendo os ansiolíticos, anti-hipertensivos e antidepressivos mais frequentemente utilizados. Os medicamentos que atuam no sistema nervoso central e no sistema cardiovascular são identificados pelos autores como um dos principais coadjuvantes das quedas, visto que podem levar a alterações psicomotoras, tonturas, hipotensão postural ou até mesmo a diminuição do fluxo sanguíneo cerebral, com perda de consciência. ${ }^{(14,24)}$

As quedas com dano, no presente estudo, representaram 50,7\% do número total de eventos, sendo, na sua maioria, de grau leve $(62,2 \%)$, seguido de moderado $(32,4 \%)$ e grave $(5,4 \%)$. A prevalência de danos decorrente de quedas encontrada em outras pesquisas brasileiras, nos remete a índices de 26 a 38,6\% ${ }^{(7,22)}$ Em um estudo espanhol realizado com 270 pacientes internados que sofreram quedas, no período de 2014 a 2016, verificou-se que 
houve algum tipo de dano em $54,2 \%$ dos pacientes. ${ }^{(25)}$ Com relação ao grau de severidade do dano, verificou-se que as quedas com dano leve foram as mais prevalentes, semelhante a outros estudos em que as escoriações/abrasões foram as lesões mais reportadas. ${ }^{(7,22,25)}$

Além disso, no presente estudo, a presença de dano moderado foi observada em $32,4 \%$ dos eventos, com a ocorrência de ferimentos cortocontusos e traumas cranioencefálicos leves. Essas lesões podem levar a intervenções como sutura e curativos, além de prolongar o tempo de internação na instituição hospitalar. Quedas que acarretaram dano moderado ao paciente foram identificadas em $6 \%$ dos eventos ocorridos em um hospital de ensino americano. ${ }^{(26)}$ As quedas associadas aos danos graves representaram 5,4\% dos eventos notificados, os quais incluíram fraturas de nariz e punho em dois pacientes. A ocorrência de fraturas em decorrência de uma queda variou de 1 a 1,6\% em outros estudos brasileiros. ${ }^{(7,22)}$ Em um estudo transversal realizado no Hospital Bundang da Universidade Nacional de Seul, na Coréia do Sul, com uma amostra de 428 quedas registradas no ano de 2015, foram reportadas $06(1,4 \%)$ quedas com diagnóstico de fratura. ${ }^{(27)}$ Ressalta-se aqui o alto índice de fraturas na pesquisa, mesmo a amostra sendo inferior às demais apresentadas, em que o índice de fraturas do presente estudo foi maior do que a média nacional e, em alguns casos, até mesmo, superior à média internacional.

Após a ocorrência da queda, o estudo enfatiza a importância de uma abordagem terapêutica segura e completa, executada por uma avaliação médica precisa e com recursos de imagem, como: raio-x e tomografias. Observou-se que apenas 17,8\% das quedas foram diagnosticadas com o auxílio do recurso de imagem. Em um estudo coreano, verificou-se que as quedas foram diagnosticadas com o auxílio de imagens de raio-x em 84 pacientes $(19,6 \%)$, imagens de tomografia computadorizada em 45 pacientes $(10,5 \%)$, ressonância magnética em um paciente $(0,2 \%)$. ${ }^{(27)}$ As condutas terapêuticas para o tratamento de quedas necessitam de protocolos seguros e de todos os recursos disponíveis para uma avaliação completa e segura.

É de extrema importância mencionar que os pacientes internados em instituições hospitalares geralmente são fragilizados e com uma condição clínica desfavorável, podendo apresentar um risco maior de agravamento das lesões causadas pelas quedas, como é o caso dos pacientes com idade avançada, várias comorbidades, vulnerabilidade social, plaquetopênicos e imunodeprimidos. Diante deste fato, é fundamental uma avaliação de risco completa dos pacientes, com auxílio de escalas preditoras validadas e adequadas à realidade institucional e ao perfil de pacientes. Nesse estudo, 82,2\% dos pacientes foram identificados para risco de queda, sendo, a maioria, classificada como risco moderado, conforme a Morse Fall Scale, porém, o uso de pulseira de identificação de risco foi observado somente em $45,2 \%$ dos pacientes e o descumprimento das orientações pelos pacientes e familiares ocorreram em $72,6 \%$ das quedas. Destaca-se aqui a necessidade de desenvolvimento de ações mais eficazes, a ponto de mitigar o evento, como a realização de uma avaliação complexa do paciente, que deverá incluir a revisão de medicamentos e pedidos de exames laboratoriais. É de suma importância, no momento da avaliação, compreender as circunstâncias que levaram o paciente a queda, isso ajuda na compreensão de causas potenciais e como evitar futuros incidentes. 


\section{Conclusão}

Os resultados deste estudo permitiram identificar as características das quedas com pacientes hospitalizados, evento este teve 73 notificações registradas entre os anos de 2016 até 2019. As mais prevalentes foram as da própria altura e ocorrida dentro dos banheiros, acometendo principalmente homens, com idade avançada, alteração de equilíbrio, em realização de tratamento clínico, além, do uso de medicações contínuas. Das quedas ocorridas, $(50,7 \%)$ geraram danos ao paciente, a maioria foi classificada como danos leves $(62,2 \%)$, destacam-se também o alto índice de descumprimento das orientações da equipe de saúde pelo próprio paciente que sofreu a queda.

Consideram-se como limitações do estudo a análise retrospectiva e o número de quedas possivelmente subnotificadas pelo hospital. Porém, é importante destacar que os resultados obtidos podem trazer contribuições para serviços de saúde, uma vez que a cultura de segurança ainda é uma temática pouco explorada no território brasileiro.

Nossos resultados sugerem a necessidade de aprimorar e até mesmo ampliar os programas existentes de prevenção de quedas nos hospitais gerais, para grupos etários específicos, estes programas devem abranger a avaliação e intervenção multidimensional do idoso, incluindo melhora da capacidade física com a realização de exercícios que envolvam treino de força, equilíbrio e marcha.

Diante desse contexto, destaca-se a importância da integralidade na assistência como um dos pilares do SUS, pois é necessário fortalecer tanto o atendimento pré-hospitalar quanto a rede de urgência e emergência, visto que consistem em portas importantes de entrada no sistema de saúde para o usuário. A assistência hospitalar, com os serviços de fisioterapia, ortopedia, neurologia e geriatria, deve ser otimizada para suprir a crescente demanda de idosos vítimas de quedas, minimizando o tempo de permanência e estimulando a deambulação e independência funcional precoce com treino de uso seguro de auxiliares para marcha, o que certamente diminuirá as complicações e número de danos. Paralelamente, os centros especializados de reabilitação devem estar preparados para absorver a demanda de idosos vítimas de quedas acidentais que necessitem de fisioterapia, terapia ocupacional e outros serviços, ou seja, a rede de cuidado à pessoa com deficiência é essencial para a integralidade na assistência para as vítimas de quedas.

O tema tem sido abordado nos últimos anos, porém, ainda carece de práticas assistenciais holísticas e preventivas. É importante que o profissional de enfermagem se aproprie e se posicione no que se refere às condutas avaliativas e educativas. Os achados colaboraram com o avanço no conhecimento científico para o ensino, a formação de novos enfermeiros e a atualização deles, chamando atenção para uma assistência que visa a segurança do paciente e a prevenção de incidentes, assim como a ampliação de novas pesquisas sobre a temática.

\section{Referências bibliográficas}

1. American Geriatrics Society; British Geriatrics Society. AGS/BGS Clinical practice guideline: for prevention of falls in older persons [Internet]. New York: AGS; 2010 [acesso em 29 set 2020]. Disponível em: www.americangeriatrics.org/health_care_ professionals/clinical_practice/clinical_guidelines_recommendations/2010

2. World Health Organization. Falls: Fact Sheet [Internet]. Genebra; 2018 [acesso em 06 set 2019]. Disponível em: http://www.who.int/mediacentre/factsheets/fs344/en/ 
3. World Health Organization: World Alliance for Patient Safety, Taxonomy: The Conceptual Framework for the International Classification for Pacient Safety: final technical report. Genebral, 2009.

4. Anderson DC,Postler TS,Dam TT. Epidemiology of hospital system patient falls: a retrospective analysis. Am J Med Qual. 2015;31(5):423-8. DOI: $10.1177 / 1062860615581199$

5. Ministério da Saúde (BR), Agência Nacional de Vigilância Sanitária. Relatórios dos Estados - eventos adversos [Internet]. 2019 [acesso em 02 dez 2019]. Disponível em: https://www20.anvisa.gov.br/segurancadopaciente/index.php/publicacoes/category/rel atorios-dos-estados

6. Falcão RMM, Vasconcelos JMN, Oliveira JS. Avaliação do risco de queda em idosos hospitalizados. Rev. Enfermagem UFPE online. 2018 Recife,12(3):812-5. DOI: 10.5205/1981-8963-v12i3a231711p812-815-2018

7. Luzia MF, Prates CG, Bombardelli CF, Adorna JB, Moura GMSS. Características das quedas com dano em pacientes hospitalizados. Rev. Gaúcha Enfermagem. 2019;40(esp):e20180307. DOI: 10.1590/1983- 1447.2019.20180307

8. Morse J. Preventing pacient falls. Thosand Oaks: Sage; 1997.

9. Urbanetto, JS et al. Morse Fall Scale: tradução e adaptação transcultural para a língua portuguesa. Revista da Escola de Enfermagem da USP [online]. 2013;47(3):569-575.

10. Stephenson M, Mcarthur A, Giles K, Lockwood C, Aromataris E, Pearson A. Prevention of falls in acute hospital settings: a multi-site audit and best practice implementation project. Int J Qual Health Care. 2016. [cited 2020 Mai 10];28(1):92-8. DOI: 10.1093/intqhe/mzv113

11. Chow SK, Lai CK, Wong TK, Suen LK, Kong SK, Chan CK, et al. Evaluation of the Morse Fall Scale: applicability in Chinese hospital populations. Int J Nurs Stud. 2007 May;44(4):556-65. DOI: 10.1016/j.ijnurstu.2005.12.003

12. Runciman W, Hibbert P, Thomson R, Van Der Schaaf T, Sherman H, Lewalle P. Towards an International Classification for Patient Safety: key concepts and terms. Int J Qual Health Care. 2009 Feb;21(1):18-26. DOI: 10.1093/intqhc/mzn057

13. Tiensoli SD, Moreira MC, Morais SM, Matozinhos FP, Gomes FSL. Contexto de quedas notificadas em um hospital universitário. Revista Baiana Enferm. 2019; 33:e32590. DOI: 10.18471/rbe.v33.32590

14. Silva AK, Costa DC, Reis AM. Fatores de risco associados às quedas intra-hospitalares notificadas ao Núcleo de Segurança do Paciente de um hospital de ensino. Einstein, São Paulo, 2019;17(1):eAO4432. DOI: 10.31744/einstein_journal/2019AO4432

15. Barbosa AS, Chaves EHB, Ribeiro RG, Quadros DV, Suzuki LM, Magalhães AMM. Caracterização dos incidentes de quedas de pacientes adultos internados em um hospital 
universitário. Rev. Gaúcha Enferm. 2019;40(esp):e20180303. DOI: 10.1590/19831447.2019.20180303

16. Sociedade Brasileira de Geriatria e Gerontologia. Quedas em idosos: Prevenção. Projeto Diretrizes. Sociedade Brasileira de Geriatria e Gerontologia; 2008.

17. Khow KSF, Visvanathan R. Falls in the aging population. Clin Geriatr Med. 2017;33(3):357-68. DOI: 10.1016/j.cger.2017.03.002

18. Gutiérrez-Robledo LM. Prevention of falls and their consequences. Eur Geriatr Med. 2016;7(6):501-2. DOI: 10.1016/j.eurger.2016.08.002

19. Klak A, Raciborski F, Targowski T, Rzodkiewicz P, Bousquet J, Samolinski B. A growing problem of falls in the aging population: a case study on Poland - 2015-2050 forecast. Eur Geriatr Med. 2017;8(2):105-10. DOI: 10.1016/j.eurger.2017.02.004

20. United Nations, Department of Economic and Social Affairs, Population Division. World population prospects: the 2017 revision, key findings and advance tables. New York: UN DESA;2017 (Working Paper $\mathrm{N}^{\circ}$ ESA/P/WP/248. Disponível em: https://reliefweb.int/sites/reliefweb.int/files/resources/WPP2017_KeyFindings.pdf

21. Pasa TS, Magnago TSBS, Urbanetto JS, Baratto MAM, Morais BX, Carollo JB. Risk assessment and incidence of falls in adult hospitalized patients. Revista Latino Amer. Enfermagem, 2017; 25:Ed.2862. DOI: 10.1590/1518-8345.1551.2862

22. Guillaume D, Crawford S, Quigley P. Characteristics of the middle-age adult inpatient fall. Appl Nurs Res. 2016;31:65-71. DOI: 10.1016/j. apnr.2016.01.003.

23. Rosa CDP, Menezes MAJ. Avaliação da influência da estrutura física das unidades de internação de clínica médica e cirúrgica de um hospital público do município de São Paulo: proposta para o gerenciamento de risco de quedas. Revista de Gestão em Sistemas de Saúde. 2015;4(1). DOI: 10.5585/rgss.v4i1.177

24. Ribeiro TB, Melo DO, Maia FOM, Ribeiro E. Medication-related inpatient falls: a critical review. Braz J Pharm Sci. 2018;54(1):e17355. DOI: 10.1590/s217597902018000117355

25. Mesas AIA, Jiménez MPC, García MVR, Vinuesa MDS, Piñero S, Pérez BM. Incidencia, factores relacionados y consecuencias de las caídas en un hospital de tercer nivel. NURE investigación: Revista Científica de Enfermería, 2018 [acesso em 06 nov 2020];15(94):4. Disponível

em: https://www.nureinvestigacion.es/OJS/index.php/nure/article/view/1363/833

26. Cox J, Hawkins CT, Pajarillo E, DeGennaro S, Cadmus E, Martinez M. Factors associated with falls in hospitalized adult patients. Appl Nurs Res. 2015;28:78-82. DOI: 10.1016/j.apnr.2014.12.003. 
27. Cho MS, Lee HY. Factors associated with injuries after inpatient falls in a Tertiary Hospital. J Korean Clin Nurs Res. 2017;23(2):202. DOI: 10.22650/JKCNR.2017.23.2.202

Participação dos autores: a) Planejamento e concepção do trabalho; b) Coleta de dados; c) Análise e interpretação de dados; d) Redação do manuscrito; e) Revisão crítica do manuscrito.

C. D. contribuiu em a, b, c, d, E; J. R. V. d. A. em d, e; M. B. d. M. em a, b, c, d, e; L. A. F. em c, d; S. C. em c, d; A. R. d. A. P. em d, e.

Editora científica responsável: Dra. Natalie Figueredo 